\title{
Solution of the generalized periodic discrete Toda equation II; Theta function solution
}

\author{
Shinsuke Iwao \\ Graduate School of Mathematical Sciences, \\ The University of Tokyo, \\ 3-8-1 Komaba Meguro-ku, Tokyo 153-8914, Japan
}

November 6, 2018

\begin{abstract}
We construct the theta function solution to the initial value problem for the generalized periodic discrete Toda equation.
\end{abstract}

\section{Introduction}

The aim of the present paper is to obtain an explicit formula for the solution to the hungry periodic discrete Toda equation (hpdToda) (1.1]1.3): $\forall n, t \in \mathbb{Z}$,

$$
\begin{aligned}
& I_{n}^{t+M}=I_{n}^{t}+V_{n}^{t}-V_{n-1}^{t+1}, \\
& V_{n}^{t+1}=\frac{I_{n+1}^{t} V_{n}^{t}}{I_{n}^{t+M}}, \\
& I_{n}^{t}=I_{n+N}^{t}, \quad V_{n}^{t}=V_{n+N}^{t},
\end{aligned}
$$

where $N$ and $M$ are positive integers. $t$ is the time variable and $n$ means the position, and relation (1.3) is just the periodic boundary condition. This system is a variant of the periodic discrete Toda equation, which is the $M=1$ case [4].

This article is a continuation of the paper [1. We will construct a tau function solution for the hungry periodic discrete Toda equation (hpdToda).

Remark: To avoid a non-interesting solution $I_{n}^{t+M}=V_{n}^{t}, V_{n}^{t+1}=I_{n+1}^{t}$, we should assume the extra constraint

$$
\prod_{n=1}^{N} I_{n}^{t+M}=\prod_{n=1}^{N} I_{n}^{t} \neq \prod_{n=1}^{N} V_{n}^{t+1}=\prod_{n=1}^{N} V_{n}^{t},
$$

which is enough to guarantee the existence of a unique solution. See theorem 2.3 . 
Notation: For a meromorphic function $f$ over a complete curve $C,(f)_{0}$ (resp. $\left.(f)_{\infty}\right)$ denotes the divisor of zeros (resp. poles) of $f$. Let $(f):=(f)_{0}-$ $(f)_{\infty} . \operatorname{Div}^{d}(C)$ means the set of divisors over $C$ of degree $d$ and $\operatorname{Pic}^{d}(C)$ means the quotient set defined by $\operatorname{Pic}^{d}(C)=\operatorname{Div}^{d}(C) /$ (linearly equivalent). For an element $\mathcal{D} \in \operatorname{Div}^{d}(C),[\mathcal{D}]$ means the image of $\mathcal{D}$ under the natural map $\operatorname{Div}^{d}(C) \rightarrow \operatorname{Pic}^{d}(C)$.

In sections 2 and 3, we consider the case g.c.d. $(N, M)=1$. We will discuss the general cases in section 4

\section{Linearization of hpdToda}

We summarize the results of [1] briefly in this section. The reader should consult the paper for further details.

\subsection{The spectral curve and the eigenvector mapping}

The hpdToda equation (1.1 1.3) is equivalent to the following matrix equation:

$$
L_{t+1}(y) R_{t+M}(y)=R_{t}(y) L_{t}(y),
$$

where $L_{t}(y)$ and $R_{t}(y)$ are given by

$$
L_{t}(y)=\left(\begin{array}{cccc}
1 & & & V_{N}^{t} \cdot 1 / y \\
V_{1}^{t} & 1 & & \\
& \ddots & \ddots & \vdots \\
& & V_{N-1}^{t} & 1
\end{array}\right), \quad R_{t}(y)=\left(\begin{array}{cccc}
I_{1}^{t} & 1 & & \\
& I_{2}^{t} & \ddots & \\
& & \ddots & 1 \\
y & & & I_{N}^{t}
\end{array}\right),
$$

and $y$ is a complex variable. Let us introduce a new matrix $X_{t}(y)$ defined by

$$
X_{t}(y):=L_{t}(y) R_{t+M-1}(y) \cdots R_{t+1}(y) R_{t}(y) .
$$

From (2.1) and (2.2), we obtain

$$
X_{t+1}(y) R_{t}(y)=R_{t}(y) X_{t}(y),
$$

which implies that the characteristic polynomial of $X_{t}(y)$ is invariant under the time evolution. Let $F(x, y):=\operatorname{det}\left(X_{t}(y)-x E\right)$ be the characteristic polynomial of $X_{t}(y)$ ( $E$ is the unit matrix). Denote the affine curve defined by $F(x, y)=0$ by $\widetilde{C}$, and its completion by $C$. Of course, $C$ is invariant as well under the time evolution. This projective curve $C$ is called the spectral curve of the hpdToda. 


\subsubsection{Properties of the spectral curve}

Now let us list the behaviour of $C$, following [1] $\S 2$.

- on $C$, there exists a point $P:(x, y)=(\infty, \infty)$ around which there exists a local coordinate $k$ such that $x=k^{-M}+\cdots$ and $y=k^{-N}+\cdots$.

- on $C$, there exists a point $Q:(x, y)=(\infty, 0)$ around which there exists a local coordinate $k$ such that $x=E k^{-1}+\cdots$ and $y=k^{N}+\cdots$, where $E=\left(\prod_{n=1}^{N} \prod_{j=0}^{M-1} I_{n}^{j}\right) \cdot \prod_{n=1}^{N} V_{n}^{0}$.

- the $M$ points $A_{j}:(x, y)=\left(0,(-1)^{N} \prod_{n} I_{n}^{j}\right), j=0,1, \ldots, M-1$ lie on $C$.

- the point $B:(x, y)=\left(0, \prod_{n} V_{n}^{t}\right)$ lies on $C$.

- The projection $p_{x}: C \ni(x, y) \mapsto x \in \mathbb{P}^{1}$ is $(M+1): 1$, and the projection $p_{y}: C \ni(x, y) \mapsto y \in \mathbb{P}^{1}$ is $N: 1$.

- $C$ has genus $g=\frac{(N-1)(M+1)-m+1}{2}$, where $m$ is the greatest common divisor of $N$ and $M$.

Hereafter we assume $C$ is smooth unless otherwise stated.

\subsubsection{The eigenvector mapping}

An isolevel set $\mathcal{T}_{C}$ is the set of matrices $X(y)$ (eq.(2.2) $)$ associated with the spectral curve $C$. Now we construct a map from $\mathcal{T}_{C}$ to $\operatorname{Pic}^{g+N-1}(C)$, called the eigenvector mapping, which plays a very important role in the present method.

Let $X=X(y)$ be an element of $\mathcal{T}_{C}$. If $(x, y) \in \widetilde{C}$, there exists a complex $N$-vector $\boldsymbol{v}(x, y)$ such that $X(y) \boldsymbol{v}(x, y)=x \boldsymbol{v}(x, y)$, up to constant multiple. Then there exists a Zariski open subset $C^{\circ}$ of $\widetilde{C}$ over which the morphism $C^{\circ} \ni(x, y) \mapsto \boldsymbol{v}(x, y) \in \mathbb{P}^{N-1}$ is uniquely determined. Moreover, for a smooth $C$, this morphism can be extended uniquely over the whole $C$. Denote this morphism by $\Psi_{X}: C \rightarrow \mathbb{P}^{N-1}$.

The eigenvector mapping $\varphi_{C}: \mathcal{T}_{C} \rightarrow \operatorname{Pic}^{d}(C)(d=g+N-1)$ is a map defined by the formula:

$$
\varphi_{C}(X)=\Psi_{X}^{*}\left(\mathcal{O}_{\mathbb{P}^{N-1}}(1)\right),
$$

where $\mathcal{O}_{\mathbb{P}^{N-1}}(1)$ is the invertible sheaf of hyperplane sections over $\mathbb{P}^{N-1}$. Note that it is nontrivial to prove $\varphi_{C}(X) \in \operatorname{Pic}^{d}(C)$ (see [1 $\S 2$ ).

The role of the eigenvector mapping is to embed the set $\mathcal{T}_{C}$ into $\operatorname{Pic}^{d}(C)$. The following proposition is originally obtained in van Moerbeke, Mumford [2].

Proposition 2.1 ([2], thm. 3) The eigenvector mapping $\varphi_{C}: \mathcal{T}_{C} \rightarrow \operatorname{Pic}^{d}(C)$ is an embedding. 
Although the definition of the eigenvector mapping is abstract, we can have an explicit formula to express $\varphi_{C}(X)$ in the present situation.

Lemma $2.2([\mathbf{1}], \S 2)$ Let $\boldsymbol{v}(x, y)=\left(\begin{array}{c}g_{1} \\ \vdots \\ g_{N}\end{array}\right)$ be an eigenvector of $X(y)$ belonging to $x\left(g_{i}=g_{i}(x, y), i=1, \ldots, N\right)$. Then it follows that $\varphi_{C}(X)=\left[\left(g_{1} / g_{N}\right)_{\infty}\right]$.

On the other hand, the divisor $\left(g_{1} / g_{N}\right)$ has the following expression ([2] prop. 1):

$$
\left(g_{1} / g_{N}\right)=\mathcal{D}_{1}+(N-1) P-\mathcal{D}_{2}-(N-1) Q,
$$

where $\mathcal{D}_{1}$ and $\mathcal{D}_{2}$ are general and positive divisors of degree $g$.

Let $\mathfrak{d}(X):=\mathcal{D}_{2}$. Lemma 2.2 is rewritten as $\varphi_{C}(X)=[\mathfrak{d}(X)+(N-1) Q]$.

\subsection{Linearization theorem}

Consider the $N \times N$ matrix $X_{t}(y)$ defined by (2.2) and the associated spectral curve $C$. Let $\sigma$ and $\tau$ be the isomorphisms on $\mathcal{T}_{C}$ defined by:

$$
\sigma\left(X_{t}(y)\right)=S X_{t}(y) S^{-1}, \quad \mu\left(X_{t}(y)\right)=R_{t}(y) X_{t}(y) R_{t}(y)^{-1}=X_{t+1}(y),
$$

where $S=\left(\begin{array}{cccc}0 & 1 & & \\ & 0 & \ddots & \\ & & \ddots & 1 \\ y & & & 0\end{array}\right)$. For the hpdToda equation (1.1]1.3, 2.1), $\sigma$ is the $n$-shift operator: $n \mapsto n+1$ and $\mu$ is the $t$-shift operator: $t \mapsto t+1$.

By calculating the divisors $\mathfrak{d}\left(\sigma\left(X_{t}\right)\right)$ and $\mathfrak{d}\left(\mu\left(X_{t}\right)\right)$, we have the following theorem which illustrates the flow of the hpdToda equation on $\operatorname{Pic}^{d}(C)$ :

Theorem 2.3 ([1]) (I): Let $\mathcal{D}$ be the divisor $\mathcal{D}=P-Q$. Then the following diagram is commutative.

$$
\begin{array}{cccc}
\mathcal{T}_{C} & \rightarrow & \operatorname{Pic}^{d}(C) \\
\sigma & \downarrow & & \downarrow \quad+[\mathcal{D}] . \\
\mathcal{T}_{C} & \rightarrow & \operatorname{Pic}^{d}(C)
\end{array}
$$

(II): Let $\mathcal{E}_{j}(j=1,2, \ldots, M)$ be the divisor $\mathcal{E}_{j}=P-A_{j}$. If $t \equiv j(\bmod M)$, the following diagram is commutative.

$$
\begin{array}{rccc} 
& \mathcal{T}_{C} & \rightarrow & \operatorname{Pic}^{d}(C) \\
\mu & \downarrow & & \downarrow \quad+\left[\mathcal{E}_{j}\right] . \\
& \mathcal{T}_{C} & \rightarrow & \operatorname{Pic}^{d}(C)
\end{array}
$$


Corollary 2.4 The time evolution $t \mapsto t+M$ is expressed as $Z \mapsto Z+[B-Q]$ on $\operatorname{Pic}^{d}(C)$.

Proof. By theorem 2.3 (II), on $\operatorname{Pic}^{d}(C),\{t \mapsto t+M\}$ is expressed by the formula: $Z \mapsto Z+\left[M P-A_{0}-A_{1}-\cdots-A_{M-1}\right]$. Then the relation $(x)=$ $-M P-Q+A_{0}+A_{1}+\cdots+A_{M-1}+B \in \operatorname{Div}^{0}(C)$ yields the result.

Corollary 2.5 The divisor $\mathcal{D}_{1}$ in 2.4 satisfies $\mathcal{D}_{1}=\mathfrak{d}\left(\sigma\left(X_{t}\right)\right)$.

Proof. By (2.4), $\left[\mathcal{D}_{1}\right]=\left[\mathfrak{d}\left(X_{t}\right)+(N-1) Q-(N-1) P\right]=\left[\mathfrak{d}\left(\sigma^{-N+1}\left(X_{t}\right)\right)\right]=$ $\left[\mathfrak{d}\left(\sigma\left(X_{t}\right)\right)\right]$. Because $\mathcal{D}_{1}$ and $\mathfrak{d}\left(\sigma\left(X_{t}\right)\right)$ are general, positive and of degree $g$, it follows that $\mathcal{D}_{1}=\mathfrak{d}\left(\sigma\left(X_{t}\right)\right)$.

Corollary 2.6 Let $\boldsymbol{v}(x, y)=\left(\begin{array}{c}g_{1} \\ \vdots \\ g_{N}\end{array}\right)$ be an eigenvector of $X(y)$ belongs to $x$.

Then (i) $\left(g_{1} / g_{N}\right)=\mathfrak{d}(\sigma X)+(N-1) P-\mathfrak{d}(X)-(N-1) Q$, and

(ii) $\left(g_{N} / y g_{N-1}\right)=\mathfrak{d}(X)+(N-1) P-\mathfrak{d}\left(\sigma^{-1} X\right)-(N-1) Q$.

Proof. Part (i) follows immediately from (2.4) and corollary 2.5, Applying (i) to the matrix $\sigma^{-1} X=S^{-1} X S$ and noticing that $S \cdot\left(g_{N} y^{-1}, g_{1}, \ldots, g_{N-1}\right)^{T}=$ $\left(g_{1}, g_{2}, \ldots, g_{N}\right)^{T}$, we obtain (ii).

Remark 2.1 The time evolution $t \mapsto t+M$ is given by the map: $\nu\left(X_{t}(y)\right):=$ $L_{t}^{-1}(y) X_{t}(y) L_{t}(y)$. In fact, 2.22.3) proves that $\nu\left(X_{t}(y)\right)=X_{t+M}(y)$.

\section{Tau function solution of the hpdToda equa- tion}

In this section, we assume g.c.d. $(N, M)=1$.

\subsection{Construction of tau functions}

We construct a theta function solution of hpdToda equation. As in the previous section, $X_{t}=X_{t}(y)$ denotes the square matrix defined by (2.2).

Let $C$ be the (smooth) spectral curve associated with $X_{t}$. Fix a symplectic basis $\alpha_{1}, \ldots, \alpha_{g} ; \beta_{1}, \ldots, \beta_{g}$ of $C$ and the normalized holomorphic differentials $\omega_{1}, \ldots, \omega_{g}$ such that $\int_{\alpha_{i}} \omega_{j}=\delta_{i, j}$. The $g \times g$ matrix $\Omega:=\left(\int_{\beta_{i}} \omega_{j}\right)_{i, j}$ is called the period matrix of $C$. For a fixed point $p_{0} \in C$, the Abel-Jacobi mapping $\boldsymbol{A}: \operatorname{Div}(C) \rightarrow \mathbb{C}^{g} /\left(\mathbb{Z}^{g}+\Omega \mathbb{Z}^{g}\right)$ is the homomorphism defined by:

$$
\sum Y_{i}-\sum Z_{j} \mapsto \sum\left(\int_{p_{0}}^{Y_{i}} \omega_{1}, \cdots, \int_{p_{0}}^{Y_{i}} \omega_{g}\right)-\sum\left(\int_{p_{0}}^{Z_{j}} \omega_{1}, \cdots, \int_{p_{0}}^{Z_{j}} \omega_{g}\right) .
$$


Let us consider the universal covering $\pi: \mathfrak{U} \rightarrow C$ and fix an inclusion $\iota$ : $C \hookrightarrow \mathfrak{U}$. For simplicity, we slightly abuse the notation " $\pi$ " and " $\iota$ " to express the derived maps $\operatorname{Div}(\mathfrak{U}) \rightarrow \operatorname{Div}(C)$ and $\operatorname{Div}(C) \hookrightarrow \operatorname{Div}(\mathfrak{U})$, respectively. Naturally, there exists a continuous lift $\widetilde{\boldsymbol{A}}: \operatorname{Div}(\mathfrak{U}) \rightarrow \mathbb{C}^{g}$ such that $\widetilde{\boldsymbol{A}} \circ \iota\left(p_{0}\right)=0$. For the projection $\rho: \mathbb{C}^{g} \rightarrow \mathbb{C}^{g} /\left(\mathbb{Z}^{g}+\Omega \mathbb{Z}^{g}\right)$, it follows that $\rho \circ \widetilde{\boldsymbol{A}}=\boldsymbol{A} \circ \pi$.

For fixed $t \in \mathbb{Z}$, assume that some lifted positive divisor $\mathfrak{D}\left(X_{t}\right) \in \operatorname{Div}^{g}(\mathfrak{U})$ with $\pi\left(\mathfrak{D}\left(X_{t}\right)\right)=\mathfrak{d}\left(X_{t}\right)$ is specified. Then there uniquely exist two positive divisors $\mathfrak{D}\left(\sigma X_{t}\right), \mathfrak{D}\left(\mu X_{t}\right) \in \operatorname{Div}^{g}(\mathfrak{U})$ such that:

$$
\begin{array}{cc}
\widetilde{\boldsymbol{A}}\left(\mathfrak{D}\left(\sigma X_{t}\right)\right)=\widetilde{\boldsymbol{A}}\left(\mathfrak{D}\left(X_{t}\right)+\iota P-\iota Q\right), & \pi\left(\mathfrak{D}\left(\sigma X_{t}\right)\right)=\mathfrak{d}\left(\sigma X_{t}\right), \\
\widetilde{\boldsymbol{A}}\left(\mathfrak{D}\left(\mu X_{t}\right)\right)=\widetilde{\boldsymbol{A}}\left(\mathfrak{D}\left(X_{t}\right)+\iota P-\iota A_{j}\right), & \pi\left(\mathfrak{D}\left(\mu X_{t}\right)\right)=\mathfrak{d}\left(\mu X_{t}\right),
\end{array}
$$

where $t \equiv j(\bmod M)$.

Let $\tau^{t}$ be a holomorphic function over $\mathfrak{U}$ defined by the formula:

$$
\tau^{t}(p)=\theta\left(\widetilde{\boldsymbol{A}}\left\{\mathfrak{D}\left(X_{t}\right)-p-\iota \Delta\right\}\right), \quad p \in \mathfrak{U},
$$

where $\theta(\bullet)=\theta(\bullet ; \Omega)$ is the Riemann theta function and $\Delta \in \operatorname{div}^{g-1}(C)$ is the theta characteristic divisor of $C$ ([3, Chap. II, cor. 3.11). To avoid cumbersome notations, we often omit the letters " $\widetilde{\boldsymbol{A}}$ ", " $\iota$ " and use a simpler expression $\tau^{t}(p)=\theta\left(\mathfrak{D}\left(X_{t}\right)-p-\Delta\right)$ when there is no confusion possible.

Although defined over $\mathfrak{U}, \tau^{t}(p)$ can also be thought of as a multi-valued holomorphic function over $C$. By the Riemann vanishing theorem (3], Chap. II, thm. 3.11), the zero divisor of $\tau^{t}(p)$ corresponds with $\mathfrak{d}\left(X_{t}\right)$.

Let $\tau_{+}^{t}(p):=\theta\left(\mathfrak{D}\left(\sigma X_{t}\right)-p-\Delta\right)$. Then, by theorem 2.3, the function

$$
\Psi^{t}(p):=\frac{\tau_{+}^{t}(p) \cdot \tau^{t+1}(p)}{\tau^{t}(p) \cdot \tau_{+}^{t+1}(p)}=\frac{\theta\left(\mathfrak{D}\left(\sigma X_{t}\right)-p-\Delta\right) \cdot \theta\left(\mathfrak{D}\left(\mu X_{t}\right)-p-\Delta\right)}{\theta\left(\mathfrak{D}\left(X_{t}\right)-p-\Delta\right) \cdot \theta\left(\mathfrak{D}\left(\mu \sigma X_{t}\right)-p-\Delta\right)}
$$

satisfies $[$ (the zeros of denominator $)]=[$ (the zeros of numerator $)] \in \operatorname{Pic}^{2 g}(C)$ and therefore, it is a single-valued and meromorphic function over $C$.

Consider an eigenvector $X_{t}(y)\left(\begin{array}{c}g_{1}^{t} \\ \vdots \\ g_{N}^{t}\end{array}\right)=x\left(\begin{array}{c}g_{1}^{t} \\ \vdots \\ g_{N}^{t}\end{array}\right), \quad\left(g_{i}^{t}=g_{i}^{t}(x, y)=g_{i}^{t}(p)\right)$. From the relation $\left(g_{1}^{t} / g_{N}^{t}\right)=\mathfrak{d}\left(\sigma X_{t}\right)+(N-1) P-\mathfrak{d}\left(X_{t}\right)-(N-1) Q$ (corollary 2.6) we derive the following equation by means of Liouville's theorem:

$$
\Psi^{t}(p)=c \times \frac{g_{1}^{t}(p) \cdot g_{N}^{t+1}(p)}{g_{N}^{t}(p) \cdot g_{1}^{t+1}(p)}, \quad c: \text { constant }
$$

By virtue of (3.4), we can calculate some special values of $\Psi^{t}(p)$ :

Lemma 3.1 On condition that g.c.d $(N, M)=1$, we have (i) $\Psi^{t}(P)=c$, (ii) $\Psi^{t}(Q)=c \times \frac{I_{N}^{t}}{I_{1}^{t}}$. 
Proof. The lemma is proved by an elementary calculation, which we shall give in the appendix.

Because $\theta(\mathfrak{D}(X)-\iota Q-\Delta)=\theta(\mathfrak{D}(X)+(\iota P-\iota Q)-\iota P-\Delta)=\theta(\mathfrak{D}(\sigma X)-$ $\iota P-\Delta)$, it follows that

$$
\Psi^{t}(Q)=\Psi_{+}^{t}(P), \quad \text { where } \quad \Psi_{+}^{t}(p)=\frac{\tau_{++}^{t}(p) \cdot \tau_{+}^{t+1}(p)}{\tau_{+}^{t}(p) \cdot \tau_{++}^{t+1}(p)} .
$$

Then lemma 3.1 implies $I_{1}^{t} \Psi_{+}^{t}(P)=I_{N}^{t} \Psi^{t}(P)$.

Repeating this argument for $\Psi_{+}(p)$, we also derive $I_{2}^{t} \Psi_{++}^{t}(P)=I_{1}^{t} \Psi_{+}^{t}(P)$, and inductively, we have that:

$$
I_{N}^{t} \Psi^{t}(P)=I_{1}^{t} \Psi_{+}^{t}(P)=I_{2}^{t} \Psi_{++}^{t}(P)=I_{3}^{t} \Psi_{+++}^{t}(P)=\cdots .
$$

Let $\Psi_{n}^{t}:=\Psi_{++\cdots+}^{t}(P)\left(n\right.$ " + "s). Finally we obtain the equations $\Psi_{n+N}^{t}=\Psi_{n}^{t}$ and $I_{n}^{t} \Psi_{n}^{t}=d$, where the number $d$ does not depend on $n$.

Next consider the following single-valued meromorphic function over $C$ :

$$
\Phi^{t}(p):=\frac{\tau^{t}(p) \cdot \tau^{t+M}(p)}{\tau_{+}^{t}(p) \cdot \tau_{-}^{t+M}(p)}=\frac{\theta\left(\mathfrak{D}\left(X_{t}\right)-p-\Delta\right) \cdot \theta\left(\mathfrak{D}\left(\nu X_{t}\right)-p-\Delta\right)}{\theta\left(\mathfrak{D}\left(\sigma X_{t}\right)-p-\Delta\right) \cdot \theta\left(\mathfrak{D}\left(\nu \sigma^{-1} X_{t}\right)-p-\Delta\right)} .
$$

Using corollary 2.6] and Liouville's theorem, we derive the following expression:

$$
\Phi^{t}(p)=c^{\prime} \times \frac{g_{N}^{t}(p) \cdot g_{N}^{t+M}(p)}{g_{1}^{t}(p) \cdot g_{N-1}^{t+M}(p) \cdot y}, \quad c^{\prime}: \text { constant },
$$

which again allows us to compute some special values of $\Phi^{t}(p)$.

Lemma 3.2 On condition that g.c.d $(N, M)=1$, we have (i) $\Phi^{t}(P)=c^{\prime}$, (ii) $\Phi^{t}(Q)=c^{\prime} \times \frac{V_{N-1}^{t}}{V_{N}^{t}}$.

Proof. SeeA.

Due to $\Phi^{t}(Q)=\Phi_{+}^{t}(P)$ and lemma 3.2 we have $V_{N}^{t} \Phi_{+}^{t}(P)=V_{N-1}^{t} \Phi^{t}(P)$, which implies

$$
V_{N-1}^{t} \Phi^{t}(P)=V_{N}^{t} \Phi_{+}^{t}(P)=V_{1}^{t} \Phi_{++}^{t}(P)=V_{2}^{t} \Phi_{+++}^{t}(P)=\cdots .
$$

Let $\Phi_{n-1}^{t}:=\Phi_{++\cdots+}^{t}(P)$ (n "+" s). Therefore we obtain $\Phi_{n+N}^{t}=\Phi_{n}^{t}$ and $V_{n}^{t} \Phi_{n}^{t}=d^{\prime}$, where the number $d^{\prime}$ does not depend on $n$.

Define $\tau_{-1}^{t}:=\tau^{t}(\iota P), \tau_{0}^{t}:=\tau_{+}^{t}(\iota P), \tau_{1}^{t}:=\tau_{++}^{t}(\iota P), \cdots, \tau_{n-1}^{t}:=\tau_{++\cdots+}^{t}(\iota P)$ (n "+"s). By the arguments above, $I_{n}^{t}$ and $V_{n}^{t}$ have following expressions:

$$
I_{n}^{t}=d \times \frac{\tau_{n-1}^{t} \cdot \tau_{n}^{t+1}}{\tau_{n}^{t} \cdot \tau_{n-1}^{t+1}}, \quad V_{n}^{t}=d^{\prime} \times \frac{\tau_{n+1}^{t} \cdot \tau_{n-1}^{t+M}}{\tau_{n}^{t} \cdot \tau_{n}^{t+M}} .
$$




\subsection{Solution of hpdToda}

For $g$-dimensional vectors $\boldsymbol{a}$ and $\boldsymbol{b},\langle\boldsymbol{a}, \boldsymbol{b}\rangle$ denotes $\boldsymbol{a}^{T} \boldsymbol{b} \in \mathbb{C}$.

By periodicity $\mathfrak{d}\left(\sigma^{N} X_{t}\right)=\mathfrak{d}\left(X_{t}\right)$, there exist integer vectors $\boldsymbol{n}, \boldsymbol{m} \in \mathbb{Z}^{g}$ such that $\widetilde{\boldsymbol{A}}(N(\iota P-\iota Q))=\boldsymbol{n}+\Omega \boldsymbol{m}$. Considering the definition of the Riemann theta function (see [3], §II.1, for example), we have

$$
\tau_{n+N}^{t}=\tau_{n}^{t} \times \exp (-2 \pi \mathrm{i} \cdot\langle\boldsymbol{m}, \boldsymbol{z}\rangle-\pi \mathrm{i} \cdot\langle\boldsymbol{m}, \Omega \boldsymbol{m}\rangle), \quad \mathrm{i}=\sqrt{-1},
$$

where $\boldsymbol{z}=\widetilde{\boldsymbol{A}}\left(\mathfrak{D}\left(\sigma^{n+1} X_{t}\right)-\iota P-\Delta\right)$. By (3.6), we have

$$
\begin{aligned}
& I_{1}^{t} I_{2}^{t} \cdots I_{N}^{t}=d^{N} \times \frac{\tau_{1}^{t} \cdot \tau_{N+1}^{t+1}}{\tau_{N+1}^{t} \cdot \tau_{1}^{t+1}}=d^{N} \times \exp \left(-2 \pi \mathrm{i} \cdot\left\langle\boldsymbol{m}, \widetilde{\boldsymbol{A}}\left(\iota P-\iota A_{j}\right)\right\rangle\right), \\
& V_{1}^{t} V_{2}^{t} \cdots V_{N}^{t}=d^{\prime N} \times \frac{\tau_{N+1}^{t} \cdot \tau_{0}^{t+M}}{\tau_{1}^{t} \cdot \tau_{N}^{t+M}} \\
& \quad=d^{\prime N} \times \exp \left(-2 \mathrm{i} \pi \cdot\left\langle\boldsymbol{m}, \widetilde{\boldsymbol{A}}\left(\iota A_{0}+\cdots+\iota A_{M-1}-(M-1) \iota P-\iota Q\right)\right\rangle\right),
\end{aligned}
$$

where $j \equiv t(\bmod M)$. Recall $\prod_{n} I_{n}^{t+M}=\prod_{n} I_{n}^{t}$ and $\prod_{n} V_{n}^{t+1}=\prod_{n} V_{n}^{t}$, which imply that $d$ depends on $t(\bmod M)$ and that $d^{\prime}$ is independent from $t$. Finally we obtain the conclusion:

Theorem 3.3 If g.c.d. $(N, M)=1$, (3.6 3.8) solves the hpdToda (1.1 1.3).

\section{The general cases}

In the previous sections, we have assumed that g.c.d. $(N, M)=1$. Unfortunately, the method which we have established in this paper cannot be applied in the general cases.

For example, when $N=M=2$, the characteristic polynomial of the matrix $X_{t}(y)$ (equation (2.2)) is:

$$
\operatorname{det}\left(X_{t}(y)-x E\right)=y^{2}-y\left(2 x+U_{1}\right)+x^{2}-U_{2} x+U_{3}-U_{4} y^{-1},
$$

where $U_{1}=I_{1}^{t} I_{2}^{t}+I_{1}^{t+1} I_{2}^{t+1}+V_{1}^{t} V_{2}^{t}, U_{2}=I_{1}^{t} I_{1}^{t+1}+I_{2}^{t} I_{2}^{t+1}+I_{1}^{t} V_{2}^{t}+I_{1}^{t+1} V_{1}^{t}+I_{2}^{t} V_{1}^{t}+$ $I_{2}^{t+1} V_{2}^{t}, U_{3}=I_{1}^{t} I_{2}^{t} I_{1}^{t+1} I_{2}^{t+1}+I_{1}^{t+1} I_{2}^{t+1} V_{1}^{t} V_{2}^{t}+V_{1}^{t} V_{2}^{t} I_{1}^{t} I_{2}^{t}, U_{4}=I_{1}^{t} I_{2}^{t} I_{1}^{t+1} I_{2}^{t+1} V_{1}^{t} V_{2}^{t}$. However, the hungry Toda system (1.1) 1.3) has the extra conserved quantity $I_{1}^{t}+I_{2}^{t}+I_{1}^{t+1}+I_{2}^{t+1}+V_{1}^{t}+V_{2}^{t}$, which is independent from $U_{1}, U_{2}, U_{3}$ and $U_{4}$. This means that the spectral curve does not faithfully reflect the data of the system.

For this reason, we should try to trace the problem to the case g.c.d. $(N, M)=$ 1. Denote by Toda $N, M$ the hungry Toda system (1.1) 1.3) associated with the positive integers $N$ and $M$. It is sufficient to prove the following statement.

Proposition 4.1 Define the initial values $I_{n}^{0}:=\zeta+o(\zeta),(\zeta \rightarrow \infty, \forall n)$ for some complex parameter $\zeta$, and let $\left\{I_{n}^{t}, V_{n}^{t}\right\}_{n, t}$ be a solution of $\operatorname{Toda}_{N, M}$. When $\zeta \rightarrow \infty$, the new sequence

$$
\left\{I_{n}^{k M+1}, I_{n}^{k M+2}, \ldots, I_{n}^{k M+M-1}, V_{n}^{k M+1}, V_{n}^{k M+2}, \ldots, V_{n}^{k M+M-1}\right\}_{n, k}
$$


is a solution of $\operatorname{Toda}_{N, M-1}$.

Proof. We shall prove the following:

$$
\begin{aligned}
& I_{n}^{k M+M-1}=I_{n}^{k M-1}+V_{n}^{k M-1}-V_{n-1}^{k M+1}+o(1), \\
& V_{n}^{k M+1}=\frac{I_{n+1}^{k M-1} V_{n}^{k M-1}}{I_{n}^{k M+M-1}} \cdot(1+o(1)) .
\end{aligned}
$$

By (1.1 1.3) and Remark (page 1), we have

$$
I_{n}^{t}=\zeta+o(\zeta),(\forall n) \Rightarrow\left\{\begin{array}{l}
I_{n}^{t+M}=\zeta+o(\zeta),(\forall n) \\
V_{n}^{t+1}=V_{n}^{t}+o(1),(\forall n)
\end{array} \quad(\zeta \rightarrow \infty)\right.
$$

Then, in our situation, it follows that $V_{n}^{k M+1}=V_{n}^{k M}+o(1)$ for all $k \in \mathbb{Z}_{\geq 0}$ and $n$. Using (1.1 1.3) again, we derive equations (4.14.2).

Applying proposition 4.1 repeatedly, we can trace the problem to the case g.c.d. $(N, M)=1$.

Example The hungry Toda system with $N=M=2$ can be traced to the case $N=2, M=3$.

$$
\text { Let } L_{0}:=\left(\begin{array}{cc}
1 & V_{2}^{0} y^{-1} \\
V_{1}^{0} & 1
\end{array}\right), R_{0}:=\left(\begin{array}{cc}
\zeta & 1 \\
y & \zeta
\end{array}\right), R_{1}:=\left(\begin{array}{cc}
I_{1}^{0} & 1 \\
y & I_{2}^{0}
\end{array}\right), R_{2}:=
$$
$\left(\begin{array}{cc}I_{1}^{1} & 1 \\ y & I_{2}^{1}\end{array}\right)$. Define $X_{0}:=L_{0} R_{2} R_{1} R_{0}$. The characteristic polynomial of $X_{0}$ is:

$$
\begin{aligned}
\operatorname{det}\left(X_{0}-x E\right)=- & y^{3}+y^{2}\left(\zeta^{2}+U_{1}\right)-y\left\{\left(2 \zeta+U_{5}\right) x+U_{1} \zeta^{2}+U_{3}\right\} \\
& +x^{2}-\left(U_{2} \zeta+U_{6}\right) x+U_{3} \zeta^{2}+U_{4}-U_{4} \zeta^{2} y^{-1}
\end{aligned}
$$

where $U_{5}=I_{1}^{0}+I_{2}^{0}+I_{1}^{1}+I_{2}^{1}+V_{1}^{0}+V_{2}^{0}$ and $U_{6}=I_{1}^{0} I_{1}^{1} V_{1}^{0}+I_{2}^{0} I_{2}^{1} V_{2}^{0}$. Note that $U_{5}$ is the hidden conserved quantity of $\operatorname{Toda}_{2,2}$. Let $\left\{I_{n}^{t}, V_{n}^{t}\right\}_{n, t}$ be the solution of Toda $_{2,3}$. Then the sequence

$$
\begin{gathered}
\lim _{\zeta \rightarrow \infty} I_{n}^{0}, \lim _{\zeta \rightarrow \infty} I_{n}^{1}, \lim _{\zeta \rightarrow \infty} I_{n}^{3}, \lim _{\zeta \rightarrow \infty} I_{n}^{4}, \lim _{\zeta \rightarrow \infty} I_{n}^{6}, \ldots ; \\
\lim _{\zeta \rightarrow \infty} V_{n}^{0}, \lim _{\zeta \rightarrow \infty} V_{n}^{1}, \lim _{\zeta \rightarrow \infty} V_{n}^{3}, \lim _{\zeta \rightarrow \infty} V_{n}^{4}, \lim _{\zeta \rightarrow \infty} V_{n}^{6}, \ldots
\end{gathered}
$$

solves $\operatorname{Toda}_{2,2}$.

\section{Acknowledgement}

The author is very grateful to Professor Tetsuji Tokihiro and Professor Ralph Willox for helpful comments on this paper. This work was supported by KAKENHI 09J07090. 


\section{A Proofs of lemmas}

Let $\Psi^{t}(p)$ and $\Phi^{t}(p)$ be the meromorphic functions defined in section 3 , We shall now prove lemma 3.1, 3.2. In the appendix, we assume g.c.d. $(N, M)=1$.

Denote the set of $N \times N$ matrices by $M_{N}(\mathbb{C})$ and the subset of diagonal matrices by $\Gamma \subset M_{N}(\mathbb{C})$. For a matrix $X \in M_{N}(\mathbb{C})$ and subsets $A, B \subset M_{N}(\mathbb{C})$, let $A+X:=\{a+X \mid a \in A\}, A X:=\{a X \mid a \in A\}, A+B:=\{a+b \mid a \in A, b \in B\}$ and $A B:=\{a b \mid a \in A, b \in B\}$.

For two meromorphic functions $f, g$ over $C$ and a point $p \in C, " f \sim g$ around $p$ " means $0<\lim _{z \rightarrow p}|f(z) / g(z)|<+\infty$.

Let $\left(g_{1}, g_{2}, \ldots, g_{N}\right)^{T}$ be an eigenvector of $X=X(y) \in \mathcal{T}_{C}$ belonging to an eigenvalue $x$. Then $g_{1}, \ldots, g_{N}$ are meromorphic functions over $C$. The following lemma is fundamental.

Lemma A.1 (i) Let $k$ be a local coordinate around P. Then $g_{1} / g_{N}=k^{N-1}+$ $\cdots, g_{2} / g_{N}=k^{N-2}+\cdots, \ldots, g_{N-1} / g_{N}=k+\cdots$.

(ii) Let $k$ be a local coordinate around $Q$. Then $g_{1} / g_{N} \sim k^{-N+1}, g_{2} / g_{N} \sim$ $k^{-N+2}, \ldots, g_{N-1} / g_{N} \sim k^{-1}$.

Proof. (i) Recall that we have $x=k^{-M}+\cdots$ and $y=k^{-N}+\cdots$ around $P$. By (2.2), $X_{t}$ is contained in the subset $\left(E+\Gamma S^{-1}\right)(\Gamma+S)^{M}=\Gamma S^{-1}+\Gamma+\Gamma S+$ $\cdots+\Gamma S^{M-1}+S^{M}$. Then the equation $X_{t}(y) \boldsymbol{v}=x \boldsymbol{v}$ implies:

$$
\left(\gamma_{-1} S^{-1}+\gamma_{0}+\gamma_{1} S+\cdots+\gamma_{M-1} S^{M-1}+S^{M}\right) \cdot \boldsymbol{v}=k^{-M} \boldsymbol{v}+\text { (higher terms), }
$$

where $\gamma_{i}(i=-1,0, \ldots, M-1)$ are diagonal matrices. Let $T:=k S$. Therefore we obtain $\left(T^{M}+\sum_{i=-1}^{M-1} k^{M-i} \gamma_{i} T^{i}\right) \cdot \boldsymbol{v}=\boldsymbol{v}+$ (higher). Because $N$ and $M$ are relatively prime, the solution of $T \boldsymbol{v}=\boldsymbol{v}$ is $\boldsymbol{v}=\left(k^{N-1}, k^{N-2}, \ldots, 1\right)^{T}$ up to a constant multiple. This fact leads to the desired result.

(ii) Let $k$ be a local coordinate around $Q$ such that $x=E k^{-1}+\cdots$ and $y=$ $k^{M}+\cdots$ (Section 2). It follows that

$$
\left(\gamma_{-1} S^{-1}+\gamma_{0}+\gamma_{1} S+\cdots+\gamma_{M-1} S^{M-1}+S^{M}\right) \cdot \boldsymbol{v}=E k^{-1} \boldsymbol{v}+\text { (higher). }
$$

Let $U:=k^{-1} S$. Then we have $\left(\gamma_{-1} U^{-1}+\sum_{i=0}^{M} k^{i+1} \gamma_{i} U^{i}\right) \cdot \boldsymbol{v}=E \boldsymbol{v}+($ higher $)$. Standard results from linear algebra prove that there exist $(N-1)$ complex numbers $c_{1}, \ldots, c_{N-1}$ such that

$$
U \cdot\left(c_{1} k^{-N+1}, c_{2} k^{-N+2}, \ldots, 1\right)^{T}=E \cdot\left(c_{1} k^{-N+1}, c_{2} k^{-N+2}, \ldots, 1\right)^{T},
$$

which leads to the desired result. 


\section{Proof of lemma 3.1}

The equation $X_{t+1}(y) R_{t}(y)=R_{t}(y) X_{t}(y)$ (2.1) implies $\left(g_{1}^{t+1}, g_{2}^{t+1} \ldots, g_{N}^{t+1}\right)=$ $R_{t}(y) \cdot\left(g_{1}^{t}, g_{2}^{t} \ldots, g_{N}^{t}\right)$. Then (3.4) gives rise to

$$
\Psi^{t}(p)=c \times \frac{g_{1}^{t}}{g_{N}^{t}} \cdot \frac{I_{N}^{t} g_{N}^{t}+g_{1}^{t} y}{I_{1}^{t} g_{1}^{t}+g_{2}^{t}} .
$$

By lemma A.1 $\Psi^{t}$ satisfies $\Psi^{t}=c+\cdots$, around $P$, and $\Psi^{t}=c \cdot\left(I_{N}^{t} / I_{1}^{t}\right)+\cdots$, around $Q$.

\section{Proof of lemma 3.2}

As mentioned in remark 2.1 one has that $L_{t}(y) X_{t+M}(y)=X_{t}(y) L_{t}(y)$, which implies $\left(g_{1}^{t}, g_{2}^{t} \ldots, g_{N}^{t}\right)=L_{t}(y) \cdot\left(g_{1}^{t+M}, g_{2}^{t+M} \ldots, g_{N}^{t+M}\right)$. Then (3.5) leads

$$
\Phi^{t}(p)=c^{\prime} \times \frac{V_{N-1}^{t} g_{N-1}^{t+M}+g_{N}^{t+M}}{V_{N}^{t} g_{N}^{t+M} y^{-1}+g_{1}^{t+M}} \cdot \frac{g_{N}^{t+M}}{g_{N-1}^{t+M} \cdot y} .
$$

By lemma A.1 $\Phi^{t}$ satisfies $\Phi^{t}=c^{\prime}+\cdots$, around $P$, and $\Phi^{t}=c^{\prime} \cdot\left(V_{N-1}^{t} / V_{N}^{t}\right)+\cdots$, around $Q$.

\section{References}

[1] Iwao S 2008 J. Phys. A. Math. Theor. 41115201

[2] van Moerbeke P and Mumford D 1979 Acta Math. 143 (1-2) 94-154

[3] Mumford D, Musili C Nori M, Previato E and Stillman M 1983 Tata Lectures on Theta I (Progress in mathematics; v.28) ed. Bass H, Oesterlé J and Weinstein A (Berlin: Birkhäuser)

[4] Tokihiro T, Nagai A and Satsuma J 1999 Inverse Problems 15 (6) 1639-62 